\title{
Rolling Loads: a New Graphical Method.
}

\author{
By Dr R. F. Muirhead.
}

\section{(Read 8th March 1918. Received 9th April 1918.)}

1. For determining the greatest bending moment produced by a rolling load consisting of two concentrated loads at a fixed distance from one another passing over a span, the usual method involves somewhat intricate algebraic discussion. Even for two concentrated loads the discussion is complicated, and for three it would be still more so.

The method here explained, which applies directly to the case when the whole of the rolling load is on the span, uses the shearingforce curve only, and gives a very simple geometrical construction for finding the position and magnitude of the greatest B.M.

We will begin by stating the construction, and then proceed to give a geometrical proof.

2. In Fig. I the stepped curve $S Z$ is the shearing force diagram for a system of four concentrated loads at fixed distances from one another, and $G G^{\prime}$ the ordinate passing through the centre of gravity of the system, and therefore making the shaded areas $A_{1}$ and $A_{2}$ equal to one another. $I I$ is the mid point of $G G^{\prime}$, the rectangle $A B B^{\prime} A^{\prime}$ has $I l$ for its middle point, and $A B=l$ the length of the span. $D H E$ parallel to $A B$ bisects the rectangle, and a line through $H$ parallel to $A^{\prime} E$ cuts the shear-curve in the points $N_{1}, N_{2}, N_{3}$.

As will be proved later, we may conclude that the greatest B.M. must occur in the section directly below that element of the load system which corresponds to one of the three points $N_{1}, N_{2}, N_{3}$; and further that it will occur below that which has the greatest positive area included between the ordinate $G G^{\prime}$, the line $N H$, and the part of the shear-curve between $N$ and $K$, where $K$ is the point of intersection of $G G^{\prime}$ with the shear-curve. The area is to be reckoned positive if the circuit bounding it is 
anti-clock when taken in the direction $K H N \ldots$ Thus in the figure, associated with the point $N_{1}$, we have the positive area $K H N_{1} T_{2} K$.

Associated with $N_{2}$ we have the negative area $K H N_{2}$, " $\quad N_{3}, \quad, \quad, \quad$ area $H K N_{2} S_{2} N_{3} H$, which is made up of a larger negative part $H K N_{2} H$ and a smaller positive part $N_{2} S_{2} N_{3} N_{2}$.

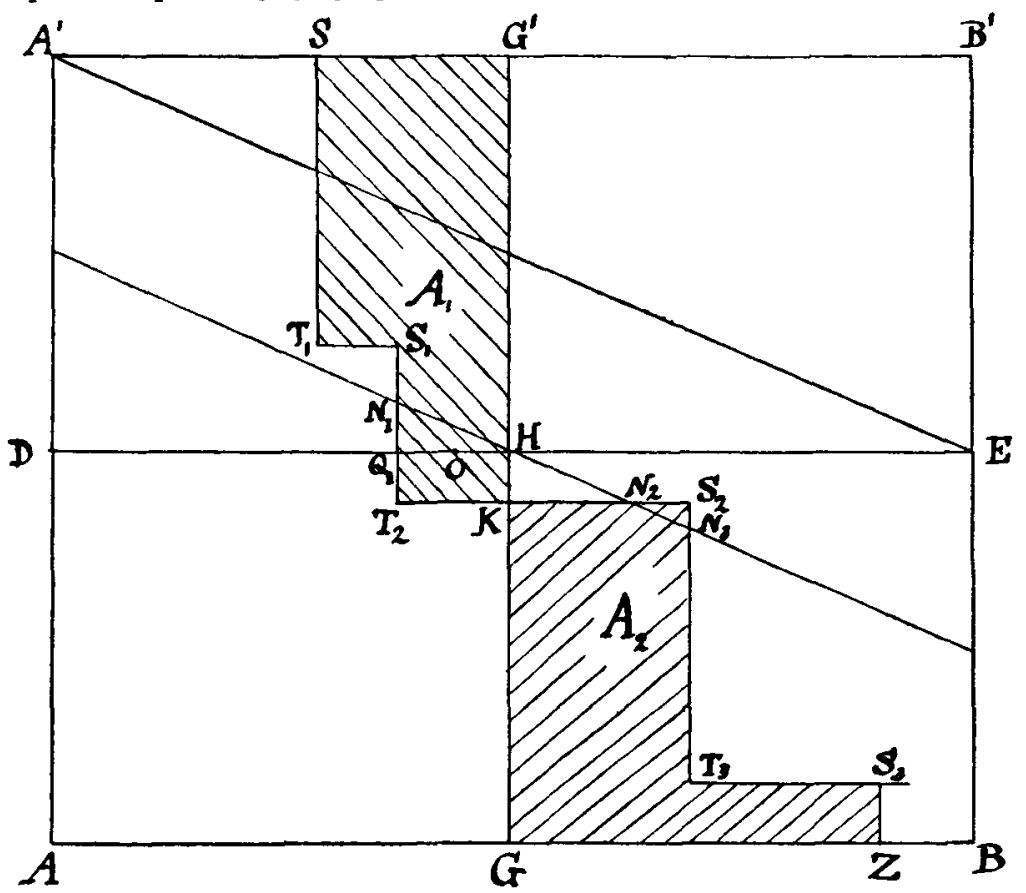

Fig. 1.

It follows that $N_{1}$ is the point of the shear-curve which has the greatest B.M. below it, i.e., the load-element $S_{1} T_{2}$ has in a certain position on the span, the greatest B.M. in the section directly beneath it, and that position is such that $O$ the mid point of the span is half-way between that element of the load and the centre of gravity of the whole load.

The amount of this greatest B.M. is given by the area $H G B E-$ Area $A_{2}+K H N_{1} T_{2} K$ on the same scale as that in which $H G B E$ would give the moment of half the load at a distance of half the span. 
3. The case of a rolling load-system with continuously varying load is illustrated in Fig. 2.

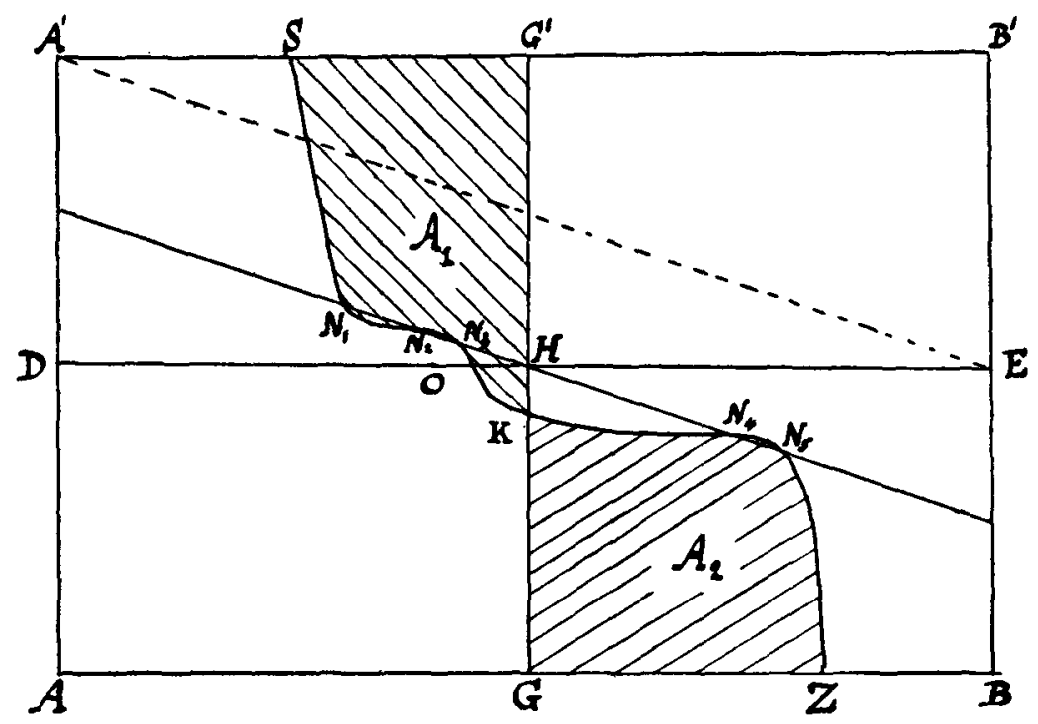

Fig. 2.

Here the letters have the same signification as in Fig. 1. The shear-curve $S Z$ is cut by the line through $I I$, parallel to $A^{\prime} E$, in $N_{1} N_{2} N_{3} N_{4} N_{5}$, giving 5 load-elements beneath which it is possible for the greatest B.M. to occur.

The area associated with the point $N_{3}$ is the positive area

$K H N_{3} K$.

\begin{tabular}{|c|c|c|c|c|c|c|c|}
\hline " & " & " & $"$ & " & " & $N_{2}$ & $\begin{array}{l}\text { consists of } K H N_{3} K \text {, } \\
\text { together with a small } \\
\text { negative area between } \\
N_{2} \text { and } N_{3} \text {. }\end{array}$ \\
\hline$"$ & $"$ & $"$ & $"$ & " & $"$ & $N_{1}$ & $\begin{array}{l}\text { consists of } K H N_{3} K, \\
\text { together with a small } \\
\text { negative area between } \\
N_{3} \text { and } N_{2} \text { and a larger } \\
\text { positive area between } \\
N_{2} \text { and } N_{1} \text {. }\end{array}$ \\
\hline
\end{tabular}


The area associated with the point $N_{\downarrow}$ is the negative area

$$
\mathrm{KHN}_{4} \mathrm{~K} \text {. }
$$

" " " " " " $\quad N_{5}$ consists of $K H N_{4} K$ and a smaller positive area between $N_{4}$ and $N_{5}$.

Thus on the whole the point $N_{1}$ has the greatest positive area associated with it, and the greatest B.M. occurring beneath it will be represented by the area $G B E H$-area $A_{2}+$ area $K H N_{1} N_{2} N_{3} K$, and will occur when $O$ the mid-point of the span is mid-way horizontally between $N_{1}$ and $H$.

In most practical cases, where the lines $H N$ cut the shearcurve at more points than one, that which gives the greatest B.M. will be easily noted by simple inspection.

It is to be noted that the above construction only applies when the whole of the load-system is on the span at the position above determined. If $O H$ were greater than $A^{\prime} S$ or $Z B$ this condition would be violated. The modification required in such a case may be explained in a later article.

4. We now proceed to give a geometrical proof of what has been stated in the foregoing explanation.

The following geometrical Lemma will be required. If $D$ is the mid-point of $B C$, a side of the triangle $A B C$, and $P M A N$ a parallelogram as shown, then the area of $P M A N$ is less than half that of $A B C$ by twice the area of the triangle $P E D$, where $D E$ drawn parallel to $B A$ meets $P M$ in $E$.

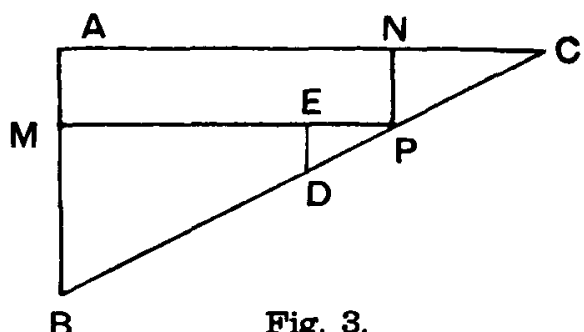

B

Fig. 3.

Corollary. It follows that the maximum area of PMAN will occur when $P$ coincides with $D$. 
5. In Fig. 4 let $A B$ represent the span, and $C$ its mid-point, and let $A^{\prime} A$ represent the total load, and $S Z$ the shear diagram for one position of the load.

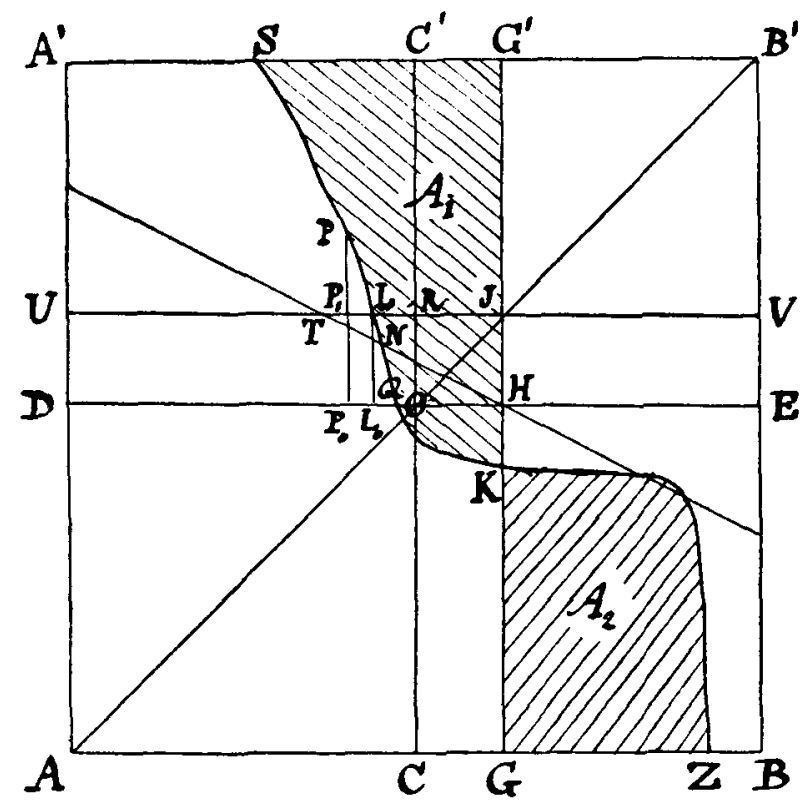

Fig. 4.

Complete the rectangle $A^{\prime} A B B^{\prime}$ and let $O$ be the mid-point of its diagonal $A C^{\prime}$, and let $D O E$ be parallel to $A B$ and $C O C^{\prime}$ parallel to $A A^{\prime}$, and let $D E$ cut the shear-curve in $Q$.

Let $G G^{\prime}$, the vertical through the centre of gravity of the load, cut $A B$ in $J$ and the shear-curve in $K$.

Then $U J V$ parallel to $A B$ will be the axis of the shear-curve for the position shown, since the areas of the rectangles $J G B V$ and $J G^{\prime} A^{\prime} U$, which represent the moments about $G$ of the supporting forces $B V$ and $U^{\prime} A$ respectively, are equal, so that the line of action of the resultant of these two supporting forces coincides with $G G^{\prime}$, as it ought.

If $P$ be any point of the shear-curve, it is obvious that the B.M. in the section to which it refers is represented by the area $U P_{1} P S A^{\prime}$. 
Now we have the following area-equivalences

$U P_{1} P S A^{\prime}-U J G^{\prime} A^{\prime}-\left(\right.$ part of area $A_{1}$ above $U V-P P_{1} L$ where $L$ is the intersection of $U V$ with the shear-curve),

$=A^{\prime} D O C^{\prime}-R O H J-A_{1}+L Q K J-P P_{1} L$ (by the Lemna),

$=\frac{1}{4} W l-A_{1}+L Q O R+Q K H-P P_{1} L$, $=\frac{1}{4} W l-A_{1}+Q K H+P_{1} P_{0} O R-P P_{0} Q L P$.

Now $A_{1}$ is a constant as well as $\frac{1}{4} W l$, and the areas $Q K H$ and $P_{1} P_{0} Q L P$ are independent of the position of the load on the span. Hence in order that the B.M. in the section directly below the chosen load-element $P$ may attain its maximum, the area $P_{1} P_{0} O R$ must be a maximum; which, by the Corollary to the Lemma, will be when $O$ bisects the horizontal distance between $P$ and $H$.

Thus we have arrived at the well-known theorem that the B.M. under any load-element attains its maximum when the mid-point of the span bisects the distance between this element and the centre of gravity of the load-system.

6. Now if the element in question has its greatest B.M. below it when $P$ is not on the shear-axis $U V$, then obviously its maximum B.M. is not the greatest, for in that position of the load there will be other elements which have a greater B.M. below them, in particular the element at which the shear is zero. Hence in searching for the greatest B.M. we need only consider such load-elements as have maximum B.M. beneath them at the instant when thershear beneath them is zero.

But this in the diagram implies that $P$ should coincide with $L$ at the same instant as $L R=R J$, and hence that $P H$ should be parallel to $A^{\prime} E$.

Thus if we draw through $H$ a line parallel to $A^{\prime} E$ meeting the shear-curve in $N$, the greatest B.M. must occur beneath the load-element corresponding to $N$ (or, in the case where there are several intersections, to one of these points of intersection).

(To avoid misconception, note that in Figs. 1 and $2 A B$ is not the span, but a length equal to the span with $G$ as mid-point, whereas in Fig. $4 A B$ represents the span itself.) 
7. If the line $H N$ meets $U V$ in $T$, obviously we have area $T H . J=$ area $R O H J$, hence we get another graphical representation of the B.M. occurring below any load-element $P$.

We have seen that it is given by

This is

$$
\begin{aligned}
& \frac{1}{4} W l-A_{1}-R O H J+L Q K J-P P_{1} L . \\
= & \frac{1}{4} W l-A_{1}-H J T+L Q K J-P P_{1} L \\
= & \frac{1}{4} W l-A_{1}+N Q K H-N L T-P P_{1} L .
\end{aligned}
$$

Thus the only variable parts are the two last terms - NLT and - $P P_{1} L$, and these are both zero when $P$ and $N$ coincide, so that for such a point the maximum B.M. is given by $\frac{1}{4} W l-A_{1}+N Q K I I$. This completes the proof of what was stated in paragraphs 2 and 3. 